Meine Versuche wurden zunächst im Anschluss an diejenigen von G. Haberlandt mit (ca. 1300) Keimlingen von Helianthus annuus $\left(96-98 \%{ }^{1}\right.$ ) , ferner mit den gross- und kleinsamigen Varietäten derselben Gattung: macrophyllus und argyrophyllus und Hel. globosus (je 50-60\%) ausgeführt. Nachdem die Ueberzeugung gewonnen war, dass bei diesen Pflanzen die Nutation durch äussere Einflüsse zwar modificirt, das Zustandekommen derselben jedoch spontan sei, wurden die Versuche auch auf andere, verschiedenen Familien angehörige Pflanzen ausgedehnt. Zunächst waren es Keimlinge von

I. $\left.{ }^{2}\right)$ Ceratonia Siliqua L. $(50-55 \%)$, Linum usitatissimum (97\%), Cynara Scolymus L. und Cynara Cardunculus (je 96\%), Pyrus Malus L. und communis L. (je 63\%), Cacurbita Pepo L. (ca. $70 \%$ ) und Cucumis sativus L. (95-98\%); ferner Cirsium, Centaurea, Aster, Viola tricolor (ca. 62\%), Ricinus communis L., Rheum, Carum Carvi L., von Coniferen (ca. 60\%): Pinus silvestris, P. Laricio, Abies excelsa und Thuja.

II. Iberis amara, Mirabilis, Cannabis sativa, Convolvulus, Raphanus sativus, Soja hispida, Phaseolus vulgaris und Ph. multiflorus.

Die Samen wurden in der Regel durch 12-24 Stunden in reinem Wasser dem Aufquellen überlassen und sodann, was, um Wiederholungen zu vermeiden, gleich jetzt bemerkt werden soll, immer, welche Lage denselben auch-gegeben wurde, nur zur Hälfte oder höchstens zu drei Viertel ihrer Länge in die Erde versenkt, einerseits, um ihnen die gewünschte Lage genauer geben zu können, andererseits, um den Druck einer darüberlastenden Erdschichte möglichst zu vermeiden.

\title{
I. Einfluss der Lage des Samens auf die Nutationsebene des Keimlings.
}

Erster Versuch.

Aus normal (d. h. vertical, mil der Wurzelspitze nach abwärts) zur Hälfte in die Erde versenkten Früchten sich entwickelnde Keimlinge von Helianthus annuus nutiren schon im Boden, und es ist die Kraft, mit welcher die Nutation vor sich geht, nicht unbe-

1) Die Angaben in \% beziehen sich auf die Keimfähigkeit der Samen der betreffenden Pflanzen; von den übrigen wurde in der Regel nur eine geringere Zahl $(30-40)$ untersucht, so dass genaue Angaben nicht geliefert werden können.

2) Der Bau der in die Gruppe I gehörigen Samen entspricht im Allgemeinen dem von Helianthus annurs, wo der Embryo gerade ist; bei den Samen der Gruppe II zeigt der Embryo eine Krümmung, er nutirt daher schon im Samen. Die Anordnung ist übrigens nebensächlich und nur der Kürze halber gewählt, da später bei Beschreibung der einzelnen Versuche darauf verwiesen wird. 
deutend, da die Samen bei Beginn der Nulationslirümmung die Erde bis auf $2^{\mathrm{mm}}$ vor sich wegdrängen, so dass hinter denselben eine Vertiefung im Boden entsteht. Dass es das blosse Gewicht der Kotylen und des Perikarps bewirke, ist wohl umsoweniger anzunehmen, als ebenso gepflanzte, doch nur mit dem oberen Viertel hervorstehende Samen sich genau so verhalten. Die Nutationsebene steht bei allen untersuchten Pflanzen in keiner Beziehung zur Mediane des Keimlings, sondern kann mit derselben alle möglichen Winkel einschliessen. Bei Helianthus und vielen anderen Keimlingen ist es Regel, dass die Nutationsebene auf der Mediane des Keimlings senkrecht steht; denn von 429 diessbezüglich untersuchten Keimlingen von Hel. annuus fiel nur bei 87 die Nutationsebene mit der Mediane zusammen, stand dagegen bei 277 auf derselben senkrecht und schloss bei 65 Keimlingen mit derselben einen schiefen Winkel ein. An geraden Keimlingen ohne jegliche Nutationskrümmung konnte ich trotz aller Vorsicht von allen 1300 Samen nur zwei erhalten, von denen der eine erst am dritten Tage zu nutiren begann. Nach.stehende Tabelle zeigt hinreichend, wie gering die Beziehung zwischen der Nutationsebene und der Mediane bei verschiedenen Pflanzen ist und wie sogar die Nutation senkrecht auf die Mediane bei manchen Pflanzen vorwaltet.

\begin{tabular}{|c|c|c|c|c|c|}
\hline \multirow{3}{*}{\multicolumn{2}{|c|}{$\underset{\text { von }}{\text { Keimlinge }}$}} & & \multicolumn{3}{|c|}{ Nutationsebene } \\
\hline & & & $\begin{array}{l}\text { in der } \\
\text { Mediane }\end{array}$ & $\begin{array}{c}\text { senkrecht } \\
\text { a. d. Mediane }\end{array}$ & schief \\
\hline & & & \multicolumn{3}{|c|}{ Procente } \\
\hline Helianthus annurs & & . . & $20 \cdot 23$ & $64 \cdot 65$ & $1 . ّ \cdot 11$ \\
\hline Hel. maerophyllus . & . & . . & $28 \cdot 94$ & $60 \cdot 52$ & $10 \cdot 53$ \\
\hline Hel. argyrophyllus & . & . . & 24 & 56 & 20 \\
\hline Ceratonia Siliqua.. & . & . . . & 26 & ธ้6 & 18 \\
\hline Cucumis sativus . . . & . & . . . & 21 & $6 \stackrel{5}{\circ}$ & 14 \\
\hline Cucurbita Pepo... & . & . . & 19 & 66 & 13 \\
\hline Viola tricolor. . . & . & . . & 34 & 51 & 17 \\
\hline Linum usitat. . . . & - & . . & 36 & 44 & 20 \\
\hline Cymara Scolymus. . & 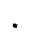 & . . & - & 94 & 6 \\
\hline Cynara Cardunculus & $\cdot$ & . . . & - & 96 & 4 \\
\hline Pinus silvestris . . . & . & . . . & 22 & 52 & 26 \\
\hline
\end{tabular}

Der Keimungsprocess der Samen ging bei verschiedener, doch für die Dauer jedes einzelnen Versuches constanter Temperatur $\left(14-30^{\circ} \mathrm{C}\right.$.) vor sich. Der einzige sich dabei ergebende Unterschied bestand in einem bedeutend rascheren Verlanfe der Nutation bei höherer Temperatur, indem sowohl Schlingenbildung als deren Auflösen bei $25-30^{\circ} \mathrm{C}$. um $3-5$ Tage früher eintrat.

Genau wie Helianthus annuus verhalten sich die Keimlinge der pag. 00 unter I angeführten Pflanzen. Auch die genannten Conife- 
ren lassen beim hypokotylen Stengelgliede keine Abhängigkeit der Nutationsebene von der Mediane erkennen, wenn man als letztere die Ebene des Samenflügels, oder, was dasselbe ist, die durch die beiden Längshanten des Samens sich gelegt zu denkende Ebene annimml, in welcher das Aufspringen der Samenschale bei der Keimung erfolgt und welche bei Thuja auch mit der Berührungsebene der beiden Kotylen zusammenfällt.

\section{Zweiter Versuch.}

Werden die Samen so zur Hälfte in die Erde gesteckt, dass sie mit der Horizontalen einen Winkel einschliessen, so lassen sich drei Fiille unterscheiden: entweder bildet die Mediane des Samens einen Winkel mil der Horizontalen (A), oder es ist die Mediane vertical und die darauf senkrechte Längsebene schliesst einen Winkel mit der Horizontalen ein (B), oder es bilden beide Ebenen einen schiefen Winkel mit der Horizontalen $(C)$. In allen drei Fällen hängt die Nutationsebene in hohem Grade von dem Winkel ab, den die Samen mit der Horizontalen einschliessen. Durch die schiefe Lage wird nach dem Hervorbrechen des Würzelchens, welches sogleich geotrop. nach abwärls wächst, unter den Kotylen eine Krümmung erzeugt, welche dem Orte nach genau mit der Nutationskrümmung übereinstimmt und deren Grösse durch den Winkel des Samens mit der Verticalen gegeben ist. Bald darauf, wenn das hypokot. Stengelglied eine Länge von kaum $1^{\mathrm{mm}}$ erreicht hat, stellt sich die wahre Nutation ein. Liegt nun der Winkel des Samens mit der Horizontalen annähernd zwischen 60 und $90^{\circ}$, so kann die Nutationskraft, welche, wio wir später hören werden, mit einem Minimum beginnt und fortwährend wächst, wenn sie nicht im Sinne der vorhandenen Krümmung wirkt, dieselbe in vielen Fällen noch überwinden und sogar eine entgegengesetzte bewerkstelligen. Diess ist jedoch nicht mehr der Fall, wenn der Winkel kleiner ist; da scheint die Nutationskraft zur Ueberwindung der vorhandenen Krümmung zu schwach zu sein und es bleibt nun in der Regel bei der künstlich eingeleiteten Krümmung, deren Verlauf der normalen Nutation genau entspricht, da es auch hier ebenso häufig zu Schlingenbildungen kommt. Ist der Winkel gleich Null, der Samen also horizonlal, so geht die Krümmung durchwegs in der gegebenen Richtung vor sich.

Das Gesagte soll nun mit einigen Beispielen belegt werden; die Samen waren in den Lagen $A, B . C$ gepflanzt. Wiederholungen der Versuche ergaben nur geringe Schwankungen, welche wohl individuellen Eigenthümlichkeiten zuzuschreiben sind. 


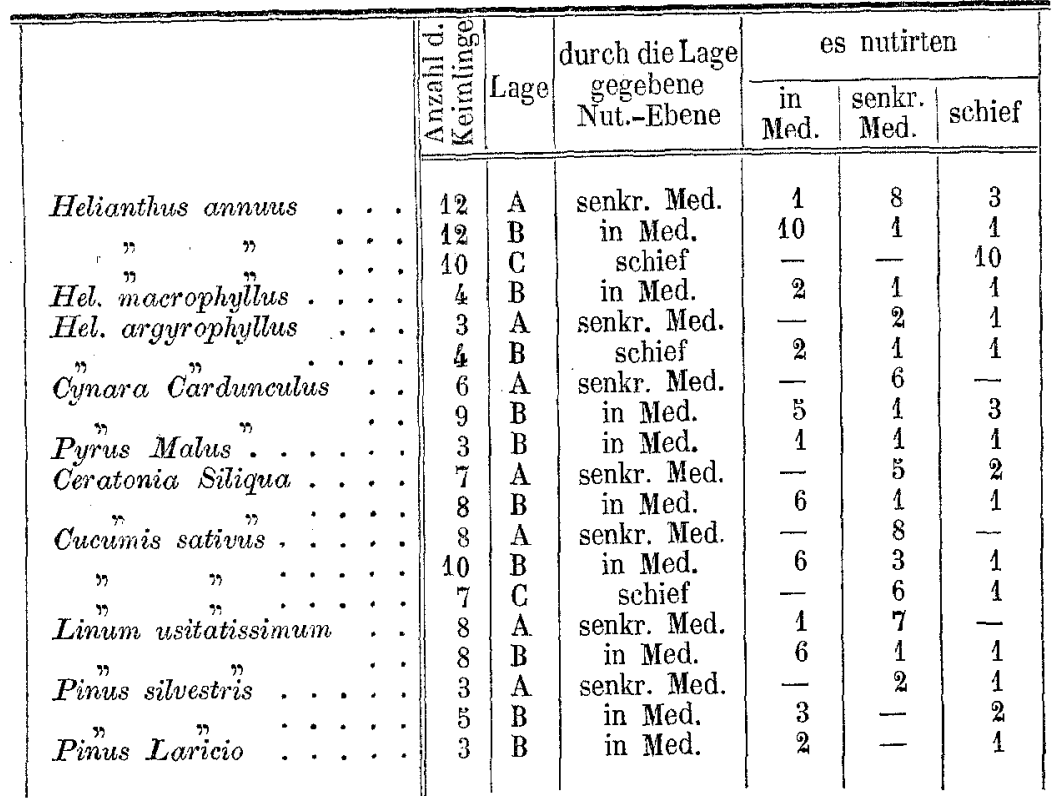

\section{Dritter Versuch.}

Derselbe unterscheidet sich von dem vorhergehenden nur bezüglich der Richlung der Keimaxe, welche hier eine entgegengesetzte war, indem die Samen in allen möglichen Lagen, doch mit der Wurzelspitze nach aufwärts gepllanzt wurden. Es liessen sich den früberen analoge Verhaltnisse wahrnehmen, doch ist es hier wieder der durch die schiefe Lage gleich nach dem Hervorbrechen des Wirzelchens zur Geltung gelangende posit. Geotropismus, welcher je nach der Lage theils hemment, theils beschleunigend wirkt. Bei verticaler Stellung der Samen wächst das hervorbrechende Würzelchen anfangs ca. $1 \mathrm{Mm}$. vertical nach aufwärts; sobald aber die Nutation eine Schielstellung desselben nach einer Seite hin eingeleitet hat, bewirkt der nun zur vollen Geltung liommende positive Geotropismus ein vertical nath abwärts Wachsen ler Wurzel. Hat sich nun der Keimling festgewurzelt, so werden die bisher noch in der Erde steckenden Kotyledonen mit dem Perikarp in Folge Längenwachsthums des hypokotylen Stengelgliedes mit einer Nutationskrümmung von $180^{\circ}$ emporgehoben. Manchmal wird aber die einmal begonnene Krummung so lebhaft fortgesetzt, dass es gar nicht zur Einwurzelung, sondern zu Doppelschlingen kommi, wobei die Kotylen mit dem Perikarp in ihrer ursprünglichen Lage zur Häfte in der Erde bleiben, was wohl auch vorzugsweise der Nachwirkung des Krummungsreizes zuzuschreiben sein dürfte. Reine Nutation ist hier nur das erste Stadium der Krümmung, die Schiefstellung des Würzelchens, wobei man sich auch hin- 
reichend von der Unabhängigkeit der Nutationsebene von der Mediane überzengen kann.

Bei schiefer Lage der Samen lassen sich, wie beim zweiten Versuche, dieselben Fälle unterscheiden; die Nutationsrichtung hängt da wieder wesentlich von der Grösse des Winkels ab. Doch tritt hier der positive Geolropismus früher, als bei verticaler Lage, gleich beim Hervorbrechen des Würzelchens auf, welches auf diese Weise auf dem kürzesten Wege in den Boden dringt und so die reine $\mathrm{Nu}-$ tation oft verdeckt wird. Nichtsdestoweniger kommt es auch vor, dass die Nutationskraft den negativen Geotropismus überwindet, indem das Würzelchen und mit ihm das hypokotyle Stengelglied nicht abwärts, sondern aufwärts wächst und erst auf der entgegengesetzten Seite in den Boden gelangt.

Wiederholungen der beiden letzteren Versuche mit Samen der Gruppe I ergaben ein gleiches Resultat; nur kam es beim dritten Versuche selten zu Schlingenbildungen, da die Keimlinge sich bald einwurzelten, und die Kotyledonen dann mit einer Nutationskrümmung von $150-180^{\circ}$ emporgehoben wurden. Keimlinge von Rheum mit einem hypokotylen Stengelgliede von $1 \mathrm{Cm}$. zeigten nach 12 Tagen (Temp. $16^{0}$ C.) weder Einwurzelung noch Schlingenbildung. Es war zwar gleich bei Beginn der Keimung eine Nutationskrümmung entstanden, welche auf ca. $90^{\circ}$ wuchs, dann aber wieder ausgeglichen wurde. Mit Coniferen wurde der dritte Versuch nicht gemacht, da die Samen derselben mit aufwarts gerichteter Wurzelspitze zar Hälfte in die Erde gesteckt nur schwer keimen.

Aus diesen Versuchen lässt sich nur auf eine immere, spontane, d. i. auf eine von äusseren Kräften unabhängige Ursache der Nułation schliessen; denn das Gewicht der Kotylen und des Perikarps kommt hier gar nicht zur Wirkung, da dieselben während der Krümmung noch im Boden befestigt sind.

\section{Einfluss künstlichen Druckes auf den Verlauf der Nutation.}

Ein vollkommen gerader Keimling von Helianthus annuus mit einem hypokotylen Stengelgliede von $7 \mathrm{Mm}$. wurde durch eine Glasplatte vou $7.5 \mathrm{~cm}$. Länge und $2.5 \mathrm{Cm}$. Breite und einem Gewichte von 5.27 Gramm seitlich derart belastet, dass dieselbe mit dem unteren Ende auf dem Boden ruhte, mit ilım einen Winkel von ca. $40^{\circ}$ einschliessend, so dass die Platte etwa in der Milte durch den gedrückten Keimling unterstützt wurde. Am nächsten Tage nutirte hei Entfernung derselben der Keimling in der Richtung des Druckes mit einem Winkel von $45^{\circ}$, welche Nutationsrichtung nachher auch beibehalten wurde. - Ein anderer Keimling mit einem hypokotylen Stengelgliede ron $1 \mathrm{Cm}$, welcher $2-3^{0}$ senkrecht auf die Mediane nutirte, wurde in derselben Weise durch 22 Stunden einem der vorhandenen Nutationsrichtung entgegen, doch in derselben Ebene wirkenden Drucke ausgesetzt. Gewicht der Glasplatte 6.78 Gramm. Der 
Keimling war in dieser Zeit um 2.5 Mm. gewachsen. Es hatte sich nun die Nutationsrichtung geändert, sie latte in eine entgegengeselzte, der Richtung des Druckes folgende umgeschlagen, und der Nutationswinkel belrug nunmehr $45^{\circ}$; der obere Theil des Keimlings hatte also einen Bogen von $47^{\circ}-48^{\circ}$ beschrieben. Sonst verlief die Nutation in der künstlichen Richlung ganz normal und trat nach 7 Tagen Schlingenbildung ein. - Ein dritter Keimling, welcher ebenfalls einen Nutationswinkel von $2^{0}-3^{0}$ zeigte, wurde in gleicher Weise einem der Nutationsrichtung entgegenwirkenden Drucke ausgesetzt, der jedoch nur 2 Stunden wahrle; es hatle die Nulationsrichtung wieder in die künstlich erzengte umgeschlagen (ca. $20^{\circ}$ ). Am nächsten Tage jedoch nutirte der Keimling nicht mehr in dieser, sondern in der früheren, urspringlichen Richtung mit einem Winkel von $10^{\circ}$, welche letztere sich auch bis zum Schlusse der Nutation $\left(180^{\circ}\right)$ erhielt.

Der auf den oberen Theil des Keimlings auszuäbende Druck lässt sich jedoch auf einfachere Art bewerkstelligen. Ein beinahe vollkommen gerader Keimling mit hypokotylem Stengelgliede von $5 \mathrm{Mm}$. wurde mit dem Finger senkrecht auf die Mediane gedrüclst, bis die Krümmung einen Winkel von $180^{\circ}$ erreicht hatte; der Druck währte 15 Min. Nach Entfernung des Fingers nahm der Winkel in Folge der Elasticität des Stengelgliedes ab und blieb auf etwa $45^{\circ}$, wuchs jedoch bis zum nächsten Tage bis $90^{\circ}$. Dasselbe Experiment wurde mit einem gleich grossen, jedoch schon merklich nutirenden $\left(3^{0}\right)$ Keimlinge durchgeführt, bis auf der der ursprünglichen entgegengesetzten Seite ein Nutationswinkel von $130^{\circ}$ erreicht war, welcher nach Entfernung des Fingers bis auf $40^{\circ}$ abnahm. Am nächsten Tage jedoch nutirte der Keimling wieder in der früheren, ursprünglichen Richtung mit einem Winkel von $30^{\circ}$. Es war also hier trotz einer künstlich erzeugten entgegengesetzten Krümmung der nutirende Theil in die ursprüngliche Richtung zurückgekehrt.

Aus diesen Versuchen geht hervor, dass es möglich ist, bei Beginn der Nutation durch künstlichen Druck jede beliebige Ebene des Keimlings zur Nutationsebene zu machen und sogar eine schon vorhandene aufzuheben, wenn nur die Nutation noch nicht zu weit vorgeschritten und der Druck ein hinreichend grosser und anhaltender ist. Ist diess nicht der Fall, so kann eine künstlich erzeugte Richtung doch wieder in die ursprüngliche zurückschlagen, welcher Umstand wohl ebenfalls auf eine spontane Nutation bei diesen Keimlingen hindeutet.

(Schluss folgt.) 